\title{
CTM Based Real-Time Queue Length Estimation at Signalized Intersection
}

Shuzhi Zhao, Shidong Liang, Huasheng Liu, and Minghui Ma

College of Transportation, Jilin University, Changchun 130022, China

Correspondence should be addressed to Huasheng Liu; liuhuasheng521@163.com

Received 28 May 2015; Revised 29 July 2015; Accepted 6 August 2015

Academic Editor: Yuanchang Xie

Copyright ( 2015 Shuzhi Zhao et al. This is an open access article distributed under the Creative Commons Attribution License, which permits unrestricted use, distribution, and reproduction in any medium, provided the original work is properly cited.

\begin{abstract}
Queue length is an important index of the efficiency of urban transport system. The traditional approaches seem insufficient for the estimation of the queue length when the traffic state fluctuates greatly. In this paper, the problem is solved by introducing the Cell Transmission Model, a macroscopic traffic flow, to describe the vehicles aggregation and discharging process at a signalized intersection. To apply the model to urban traffic appropriately, some of its rules were improved accordingly. Besides, we can estimate the density of each cell of the road in a short time interval. We, first, identify the cell, where the tail of the queue is located. Then, we calculate the exact location of the rear of the queue. The models are evaluated by comparing the estimated maximum queue length and average queue length with the results of simulation calibrated by field data and testing of queue tail trajectories. The results show that the proposed model can estimate the maximum and average queue length, as well as the real-time queue length with satisfactory accuracy.
\end{abstract}

\section{Introduction}

It has been generally recognized that the vehicular queue length is a crucial quantitative measure used in the evaluation of the performance of signalized intersections [1-3] and signal optimization [4-9]. Due to the importance of this traffic parameter, many scholars studied this topic, and their proposed models can be classified into three types: inputoutput accumulation curve methods, statistical methods, and the shockwave theory.

The first type models are based on the construction of accumulation curves by means of analysis of vehicles inputoutput to a signal link, where vehicles between two curves are accounted for as being in a queue. The method was first demonstrated by Webster [4] and later improved by many scholars [5, 10-12]. Although such models are widely used to calculate the queue length, it is hard to obtain the real-time queue length. In addition, the accumulation inputoutput curve is difficult to structure under complex traffic conditions. Therefore, such methods are limited in their ability to describe complex queuing processes.

According to statistics methods, the vehicle arrival and release processes are regarded as some certain probability distributions, where the queue length is the mathematical expectation of the difference between the numbers of arriving and released vehicles [13]. Usually, such methods are used for steady-state traffic flows, because the distribution should change in different traffic states. Commonly, it is assumed that the arrival distribution follows the Poisson distribution [14] under free flow and negative binomial distribution under saturated flow [15]. In other words, the robustness of such models is not strong enough, as they cannot adapt to the situation when traffic state changes irregularly, such as in the real world.

The shockwave theory is another powerful tool used in queue length estimation, which was proposed by Lighthill and Whitham $[16,17]$ and Richards $[18]$ to describe the queuing process of uninterrupted traffic flows on freeways. Later, Stephanopoulos et al. [19] expanded the theory for continuum traffic flows at signalized intersections. The validity of this method was proved by many scholars [20,21]. These elegant theoretical models only perform well in the situation when the traffic flow can be regarded as a steady flow macroscopically. For example, such models assume that vehicle flows transform from one state to another instantaneously. 
Given these observations, the common deficiencies of current methods can be described as their inability to obtain the real-time spatial distribution of queue lengths when traffic flows constantly change. To solve this problem and describe traffic flows at signalized intersections, a discrete macroscopic traffic flow model, the Cell Transmission Model (CTM), is introduced in this paper. The model was proposed by Daganzo [22]. This method can be used to describe traffic changes over time and space, including transient phenomena such as building, propagation, and dissipation of queues. The CTM can overcome computational difficulties caused by the substitution of difference equations for partial differential equations. The method automatically generates appropriate changes in the density at locations where the hydrodynamic theory produces shockwaves and sudden changes in the density, those typically seen at the end of a queue. Therefore, complex calculations required by classical methods are eliminated.

The CTM of motorway traffic is a discrete version of the simple continuum model of traffic flow that is convenient for computer implementations. The first order hydrodynamic model of traffic flow can be programmed using cell transmission scheme sending and receiving functions. Muñoz et al. [23] proposed a semiautomated method to calibrate the parameters of the model. According to the authors, a least-square fitting approach is applied to the loop detector data to determine free-flow and congestion speeds, as well as jam densities for specific subsections of a freeway segment. Bottleneck capacities are estimated by measuring mainline and onramp flows. Sun et al. [24] applied the mixture Kalman filtering to the CTM. Their method is able to estimate vehicle densities and congestion statuses at locations, which are not directly observed. The program runs efficiently, making it possible to carry out estimations in real time. Tampère and Immers [25] proposed another traffic estimation and prediction model based on the CTM. In their model, they provided parameters for automatic estimation of traffic conditions. Hadiuzzaman and Qiu [26] utilized the CTM in variable speed limit on freeways. They developed an analytical model based on the CTM capable of describing active bottlenecks, where the capacity drops once the incoming flow exceeds the capacity, and variable free-flow speeds in cells operated using VSL control.

Kim and Keller [27] and Pohlmann and Friedrich [28] introduced the CTM to the problem of traffic control in urban networks. They use the CTM to model the effects of different signal settings. The model is capable of adapting every 15 minutes to the currently estimated traffic and optimizing signal plans and coordination according to the demand in the network. Xie et al. [29] formalized the description of the urban CTM and evaluated its capability to model traffic volumes and jams using a microscopic traffic simulator. The CTM was further extended by Chiou and Huang [30] to describe hybrid traffic flows on urban roads. In their work, the mixed traffic CTM is proposed to replicate traffic behavior on Asian urban streets, where mixed traffic of cars and motorcycles is prevailing. The mixed CTM uses the ratio of cars to motorcycles in the last upstream cell to determine the amount of roadway resources allocated to cars and motorcycles.

Therefore, the advantages of the CTM can be summarized as follows:

(1) Satisfactory performance for description of traffic phenomena such as the queuing process and traffic wave propagation.

(2) Relative simplicity for modeling, compared to traditional traffic flow models, which is convenient for applications using computer programs.

(3) Flexibility for applications, since the traffic parameters can be estimated for both online and offline, which ensures that the queue length can be estimated in time.

The objective of this paper is to provide a strong approach to real-time queue length estimation. We focus on obtaining the maximum and average queue length within a signal cycle and tracking the queue tails trajectories. The performance of the proposed methodology partly relies on the traffic flow theory used for describing queuing processes at signalized intersections. Therefore, the CTM was tested to check its adaptation to traffic flows at signalized intersections. Accordingly, some rules were modified to adapt the original model to urban roads. Besides, the density plays an important role in queue length estimation within a discrete time interval. The density in each cell is analyzed to identify the queue tail cell and eventually calculate the exact position in this cell. It is worth mentioning that the fixed detectors of traditional signal control systems (such as SCOOT) can provide the data required by the proposed model.

The rest of the paper is organized as follows. In Section 2, we introduce the Cell Transmission Model, test it using numerical simulations, and modify it accordingly. In Section 3, we present an estimation mode of queue length and the general formula for queue length calculation. We also discuss three special cases. In Section 4, we show the testing results, including the comparison of the average and maximum queue lengths obtained in estimations and their corresponding real values, and the results on the trajectories of queue tails. Section 5 discusses some limitations of our approach and presents future work.

\section{Proposed Model for Traffic Flow Description}

The traditional Cell Transmission Model partitions a oneway road into several, say $I$, homogenous cells. The length of each cell is equal to the distance traveled by free-flowing traffic in a time interval $T$. So, in the case of free flow, all the vehicles travelling at the maximum speed can move from one cell to the next one in the unit time interval. In each cell $i$ during time period $k$, the density is approximated by $\rho_{i}(k)$, and the exit flow $q_{i}(k)$ is expressed as the minimum between the upstream demand and the downstream supply.

To describe the traffic flow at a signalized intersection, two modifications in the model are proposed. The first modification concerns the rules for the end downstream cell 


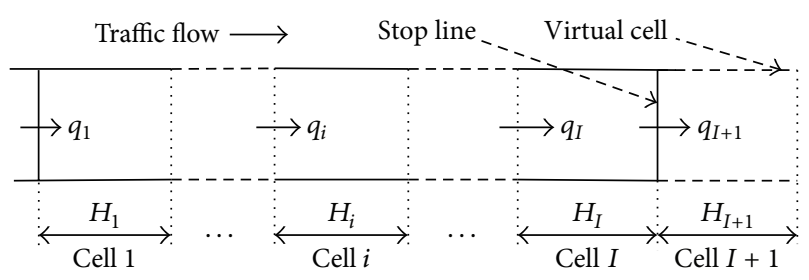

FIgURE 1: Transmission mechanism.

during the red and green phases separately. The second one modifies the rules for the discharging process of the queue based on numerical simulation analysis.

2.1. Density Dynamics. To apply the CTM in the case of traffic flows at signalized intersections, we assume that the road, from the loop detector upstream to the stop line downstream, is divided into homogeneous cells, and the length of each cell is defined as $H_{i}$. Figure 1 illustrates the vehicle transmission mechanism.

In period $k+1$, the number of vehicles in cell $i, n_{i}(k+1)$, decreases by the number of exiting vehicles and increases by the number of entering vehicles, which can be written as

$$
n_{i}(k+1)=n_{i}(k)+y_{i}(k)-y_{i+1}(k),
$$

where $y_{i}(k)$ represents the number of vehicles travelling into cell $i$ from $k$ to $k+1$. The number of vehicles in cell $i$ and travelling between two cells can be represented using basic traffic parameters, the density and traffic flow, respectively:

$$
\begin{aligned}
& n_{i}(k)=\lambda \cdot \rho_{i}(k) \cdot H_{i}, \\
& y_{i}(k)=q_{i}(k) \cdot T,
\end{aligned}
$$

where $\rho_{i}(k)$ represents the traffic density in cell $i$ for each lane, from $k-1$ to $k$; and $q_{i}(k)$ represents the traffic volume from cell $i-1$ to cell $i$ in the last time interval for each lane. Here, $\lambda$ means the number of lanes; $H_{i}$ refers to the length of the cell $v_{f} \cdot T$.

Using (1), (2), and (3), we can derive the relationship between $\rho_{i}(k)$ and $q_{i}(k)$, with the density dynamics expressed by

$$
\rho_{i}(k+1)=\rho_{i}(k)+\frac{T}{\lambda \cdot L_{i}}\left(q_{i}(k)-q_{i+1}(k)\right) .
$$

The traffic flow between two adjacent cells depends on the space in the downstream cell and demand of the upstream cell, as well as the capacity of each cell $Q_{\max }$. In the traditional CTM, traffic running in each cell is characterized by the FD shown in Figure 2. In the linear fundamental diagram, from the origin to point $A^{\prime}$, the traffic flow is free, and vehicles travel at maximum velocity. Between points $A^{\prime}$ and $A$, traffic becomes congested, and the traffic volume stays at the level of capacity, instead of increasing along with the growing density. From point $A$, the traffic flow switches to a jam, and the shockwave starts to propagate to the upstream.

The interpretation of the demand and supply functions can be illustrated as follows. If the cell density $\rho_{i}(k)$ is

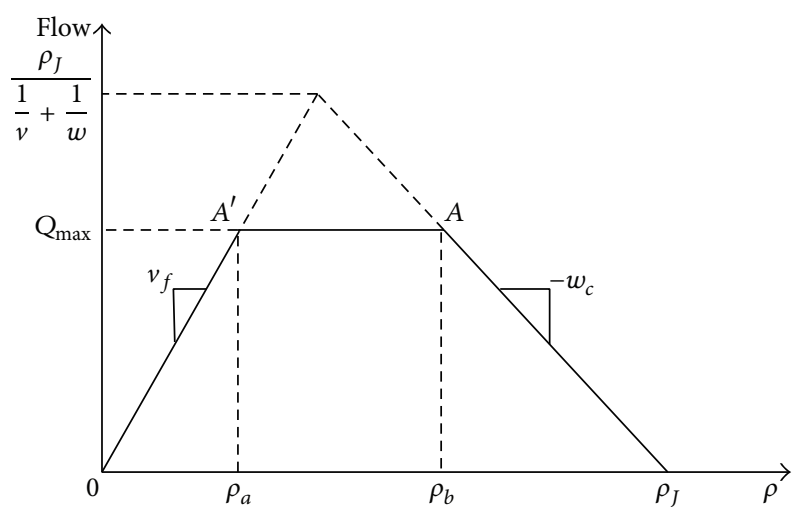

Figure 2: Fundamental diagram of the Cell Transmission Model.

lower than the density of $\rho_{a}$, cell $i$ demands space from its downstream cell $(i+1)$ for a flow of $v_{f} \cdot \rho_{i}(k)$, where $v_{f}$ means the velocity of the free flow. Otherwise, not all vehicles can travel to the downstream cell even at the saturation flow rate, because of the limitation of capacity. The demand for space from the upstream cell $(i+1)$ for a flow of $\phi_{i}(k)$ is expressed as

$$
\phi_{i}(k)= \begin{cases}\lambda \cdot v_{f} \cdot \rho_{i}(k), & \text { if } \rho_{i}(k)<\rho_{a}, \\ Q_{\max }, & \text { if } \rho_{i}(k) \geq \rho_{a} .\end{cases}
$$

If the spare space in cell $i$ is enough for the coming vehicles from cell $i-1$, it provides space to the upstream for saturated traffic flow. Alternatively, if the shockwave occurs, the supplied space will be less than the traffic capacity. The supply for the upstream cell for a flow of $\varphi_{i}(k)$ can be written as follows:

$$
\varphi_{i}(k)=\min \left\{\begin{array}{l}
Q_{\max }, \\
\lambda \cdot w_{c} \cdot\left(\rho_{J}-\rho_{i}(k)\right) .
\end{array}\right.
$$

Having the supply and demand function, we can calculate the traffic volume in those cells using the following function:

$$
\begin{aligned}
q_{i}(k) & =\min \left\{\phi_{i-1}(k), \varphi_{i}(k)\right\} \\
& =\min \left\{v_{f} \cdot \rho_{i-1}(k), Q_{\max }, w_{c} \cdot\left(\rho_{J}-\rho_{i}(k)\right)\right\} .
\end{aligned}
$$

Now, knowing the density and flux values in the given time interval, using (7), the density evolution in all cells can be estimated using (4). The proposed traffic flow model can be implemented for any number of cells in the same manner as explained above.

2.2. Output for Boundary Cells at Signalized Intersection. For finite roads, boundary conditions can be specified by means of input and output cells. The input flow can be obtained from the loop detector directly, which is discussed further in the following section on implementation. Either the data from a fixed detector or signal timing at the intersection can model the output flow. Because the data is assumed to be collected 
by the SCOOT system in this paper, there is no detector at the stop line. Therefore, another estimation method for the output cell, using signal timing parameters, is proposed.

Since in the traditional CTM signal control is not considered, we provide further analysis for the output cell during the red and green phases. When the signal light is red, none of the vehicles can pass the stop line. Therefore, the traffic flow is zero, although there can be significant supply in each cell $i+1$ due to its upstream cell $i$. The supply and demand space can be written as follows:

$$
\begin{aligned}
& \phi_{I}^{r}(k) \\
& \quad= \begin{cases}\lambda \cdot v_{f} \cdot \rho_{I}(k), & \text { if } \rho_{I}(k)<\rho_{a}, k \in \text { redphase, } \\
Q_{\max }, & \text { if } \rho_{I}(k) \geq \rho_{a}, k \in \text { redphase, }\end{cases} \\
& \varphi_{I+1}^{r}(k)=0, \quad \text { if } k \in \text { redphase, }
\end{aligned}
$$

where $\phi_{I}^{r}(k)$ means the demand space from cell $I+1$ and $\varphi_{I+1}^{r}(k)$ represents the supply space for cell $I$ when $t$ represents the red phase.

When the signal switches to green, at the beginning, the vehicles enter the intersection at the saturation flow rate. During the end of the green phase, the output flow depends on the density of the output cell. If the density is larger than its critical density, the output flow is still at the saturation rate. Otherwise, the vehicles leave the cell at the rate of $v_{f} \cdot \rho_{I}(k)$. Regarding the supply space in cell $I$, as there is no block downstream, it can be regarded as plus infinity, as shown in Figure 3. This can be expressed as follows:

$$
\begin{aligned}
& \phi_{I}^{g}(k) \\
& \quad= \begin{cases}\lambda \cdot v_{f} \cdot \rho_{I}(k), & \text { if } \rho_{I}(k)<\rho_{a}, k \in \text { greenphase, } \\
Q_{\max }, & \text { if } \rho_{I}(k) \geq \rho_{a}, k \in \text { greenphase, }\end{cases} \\
& \varphi_{I+1}^{g}(k)=+\infty, \quad \text { if } k \in \text { greenphase, }
\end{aligned}
$$

where $\phi_{I}^{g}(k)$ means the demand space from cell $I+1$ and $\varphi_{I+1}^{g}(k)$ represents the supply space in cell $I$ when $k$ is in the green phase.
Thus, the traffic flow functions for the output cell can be written as follows:

$$
\begin{aligned}
& q_{I+1}(k) \\
& = \begin{cases}\min \left\{\phi_{I}^{r}(k), \varphi_{I+1}^{r}(k)\right\} & \text { if } k \in \text { redphase } \\
\min \left\{\phi_{I}^{g}(k), \varphi_{I+1}^{g}(k)\right\} & \text { if } k \in \text { greenphase }\end{cases} \\
& = \begin{cases}0 & \text { if } k \in \text { redphase } \\
\min \left\{\lambda \cdot v_{f} \cdot \rho_{I}(k), Q_{\max }\right\} & \text { if } k \in \text { greenphase. }\end{cases}
\end{aligned}
$$

2.3. Modification of the CTM. Since the original model was developed for freeways, a numerical simulation to check its correctness in describing of vehicle aggregation at signalized intersections is necessary. To be closer to real situations, the following parameters are assumed in the simulation: a oneway road with three lanes, the maximum traffic density on road being $0.4332 \mathrm{veh} / \mathrm{m}$, the critical traffic density being $0.1 \mathrm{veh} / \mathrm{m}$, the length of each cell being $60 \mathrm{~m}$, and the number of these cells being 8 . At each time interval of 4 seconds, the density values of all cells were estimated using a Matlab program implementing the discussion above.

As shown in Table 1, the increase in density values means the aggregation of vehicles, and the decrease in density values refers to the release of vehicles. Therefore, to a certain extent, the basic CTM can describe the queue process qualitatively. However, it is noticeable that the density evolutions in cells are different from each other during the process of queue discharging. It becomes clear in Table 1, which shows the density values in each cell from 419 s to 520 s. In cell 8 , the density values changed to the critical density within $40 \mathrm{~s}$ and in cells 7, 6, and 5 within $60 \mathrm{~s}, 76 \mathrm{~s}$, and $84 \mathrm{~s}$, respectively. However, according to the assumption of the CTM, the cells of the road are homogenous, so that the density evolutions should be similar, and the time discussed above has to be approximately equal for each cell. So, the basic model should be modified to describe the queue discharging process.

The analysis of the real queue release process shows that the vehicles in the output cell leave first, and the vehicles in the immediate upstream cell cannot flow into the output cell until the density gets close to the critical density. This rule is applied to every cell up to the queue tail cell. During the queue discharging process, the traffic density in the downstream is larger than both the upstream and critical densities. The function of flow can be written as follows:

$$
q_{i}(k)= \begin{cases}0, & \text { if } \rho_{i-1}(k)>\rho_{i}(k), H_{i} \cdot \rho_{i}(k)-Q_{\max } \cdot T>\rho_{b} \\ \frac{\left(\lambda \cdot\left(2 \cdot \rho_{b}-\rho_{i}(k)\right) \cdot H_{i}\right)}{T}, & \text { if } \rho_{i-1}(k)>\rho_{i}(k), H_{i} \cdot \rho_{i}(k)-Q_{\max } \cdot T<\rho_{b} \\ \min \left\{\lambda \cdot \rho_{i}(k) v_{i}(k), Q_{\max }, \lambda \cdot\left(\rho_{j}-\rho_{i}(k)\right) \cdot H_{i}\right\}, & \text { else. }\end{cases}
$$

The density values were generated once again according to the modified model, by using a Matlab program. The results are shown in Figure 4.
As can be clearly seen in Figure 4, the slopes of contour line are similar during the queue discharging process, which means the evolutions of density in the cells are similar to each 


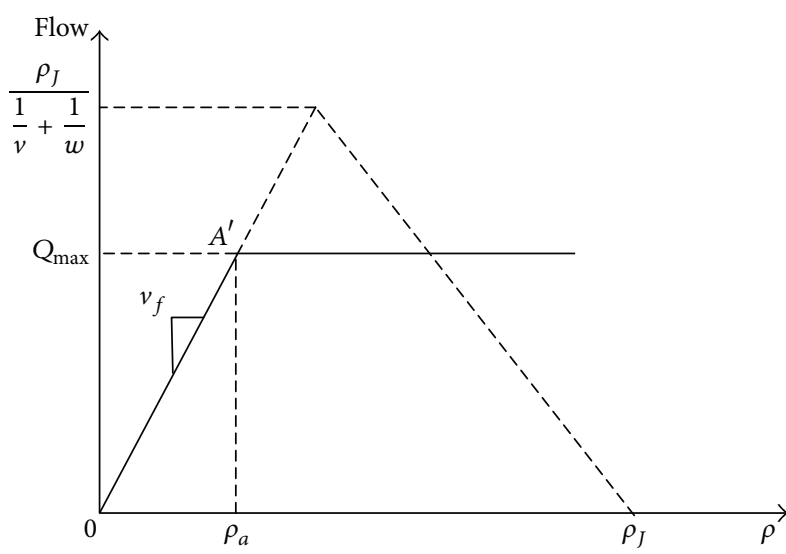

FIGURE 3: FD for cell $I+1$ during the green phase.

TABLE 1: Density values evolution based on the basic model.

\begin{tabular}{|c|c|c|c|c|c|c|c|}
\hline \multirow{2}{*}{$t(\mathrm{~s})$} & \multicolumn{7}{|c|}{ Cell } \\
\hline & 2 & 3 & 4 & 5 & 6 & 7 & 8 \\
\hline 412 & 0.1884 & 0.4332 & 0.4332 & 0.4332 & 0.4332 & 0.4332 & 0.4332 \\
\hline 416 & 0.1884 & 0.4332 & 0.4332 & 0.4332 & 0.4332 & 0.4332 & $\underline{0.3332}$ \\
\hline 420 & 0.1884 & 0.4332 & 0.4332 & 0.4332 & 0.4332 & 0.4032 & 0.2632 \\
\hline 424 & 0.1884 & 0.4332 & 0.4332 & 0.4332 & 0.4242 & 0.3612 & $\underline{0.2143}$ \\
\hline 428 & 0.1884 & 0.4332 & 0.4332 & 0.4305 & 0.4053 & & $\underline{0.18}$ \\
\hline 432 & 0.1884 & 0.4332 & 0.4324 & 0.4229 & 0.3789 & 0.276 & $\underline{0.156}$ \\
\hline 436 & 0.1884 & 0.433 & 0.4296 & 0.4097 & 0.348 & 0.24 & $\underline{0.1392}$ \\
\hline 440 & 0.1883 & 0.4319 & 0.4236 & 0.3912 & 0.3156 & 0.2098 & $\underline{0.1274}$ \\
\hline 444 & 0.1879 & 0.4294 & 0.4139 & 0.3685 & 0.2838 & & $\underline{0.1192}$ \\
\hline 448 & 0.1868 & 0.4248 & 0.4003 & 0.3431 & 0.2542 & 0.1653 & 0.1134 \\
\hline 452 & 0.1842 & 0.41 & 0.3831 & 0.3164 & 0.2275 & 0.1 & 0.1094 \\
\hline 456 & 0.1795 & 0.4 & 0.3631 & 8 & 0.2042 & 0.1 & 0.1066 \\
\hline 460 & 0.1717 & 0.3939 & 0.3411 & 0.2641 & 0.1842 & 0.1283 & 0.1046 \\
\hline 464 & 0.1599 & 0.3781 & 0.318 & 0.2401 & 0.1675 & 0.1212 & 0.1032 \\
\hline 468 & 0.1434 & 0.3601 & 0.2946 & 0.2183 & 0.1536 & 0.1158 & 0.1023 \\
\hline 472 & 0.1214 & 0.3404 & 0.2717 & 0.1989 & 0.1423 & 0.1118 & 0.1016 \\
\hline 476 & 0.0936 & 0.3198 & 0.2499 & 0.1819 & 0.1331 & 0.1087 & 0.1011 \\
\hline 480 & 0.0596 & 0.2989 & 0.2295 & 0.1673 & 0.1258 & 0.1064 & 0.1008 \\
\hline 484 & 0.0193 & 0.278 & 0.2108 & 0.1548 & 0.12 & & 0.1005 \\
\hline 488 & 0 & 0.2306 & 0.194 & 0.1444 & 0.1154 & 0.1035 & 0.1004 \\
\hline 492 & 0 & 0.1589 & 0.1791 & 0.1357 & 0.1118 & 0.1025 & 0.1003 \\
\hline 496 & 0 & 0.0827 & 0.1661 & 0.1285 & 0.109 & 0.1019 & 0.1002 \\
\hline 500 & 0 & 0.0025 & 0.1548 & 0.1227 & 0.1069 & 0.1014 & 0.1001 \\
\hline 504 & 0 & 0 & 0.0642 & 0.1179 & 0.1052 & 0.101 & 0.1001 \\
\hline 508 & 0 & 0 & 0 & 0.0837 & 0.104 & 0.1007 & 0.1001 \\
\hline 512 & 0 & 0 & 0 & 0 & 0.0879 & 0.1005 & 0.1 \\
\hline 516 & 0 & 0 & 0 & 0 & 0 & 0.0885 & 0.1 \\
\hline 520 & 0 & 0 & 0 & 0 & 0 & 0 & 0.0886 \\
\hline
\end{tabular}

other. This result is similar to some traditional traffic flow theories, which indicates that the modified model can be used to depict the discontinuous flow at intersections with signal control.

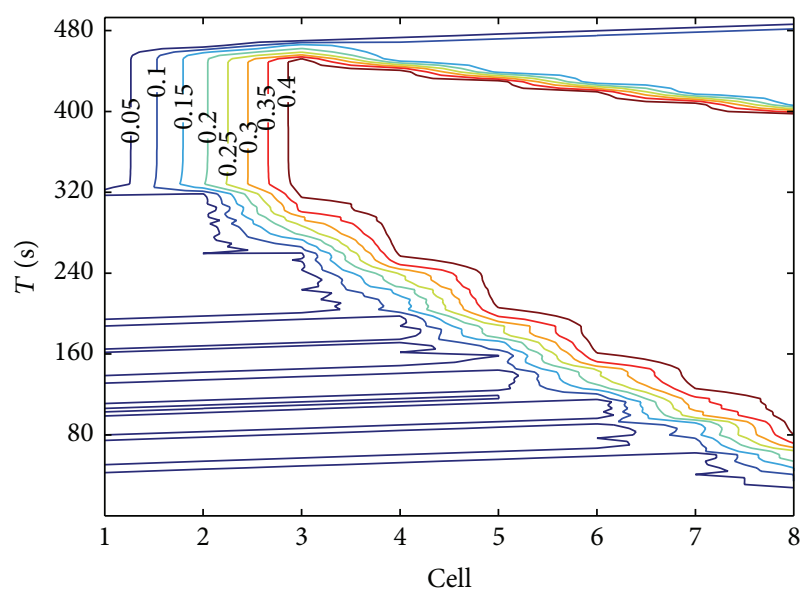

FIgURe 4: Density evolution based on the modified model.

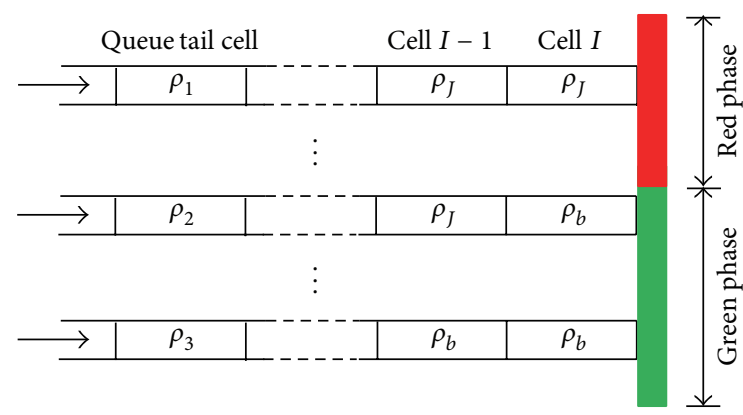

FIGURE 5: Density values evolution in a signal cycle.

\section{Queue Estimation Model}

This research proposes a real-time queue estimation model that aims to identify the point where the tail of the queue is located at every moment. Further, the maximum and average queue length can be calculated based on the realtime queue lengths. There are two steps according to the proposed method. The first step is to identify the cell where the tail of the queue is located, based on the density values in every cell. In the second step, the exact queue tail point is identified based on the density distribution in the cell selected in the first step. To overcome traffic density abrupt changes, it is assumed that in the queue tail cell the traffic density transforms into another state linearly. The formulation and analysis of this linear change are presented in Section 3.2.

3.1. Queue Tail Cell Identification. To understand the queue discharging process, a typical cycle of the evolution of cell density is shown in Figure 5. Similar processes are repeated in all cycles.

The queue starts to discharge at the beginning of the green phase, and the densities of cells between the stop line and queue tail are equal to the jam density. While discharging, some densities in those downstream cells become critical, while other upstream cells are still under the jam condition. Sometime after the green light started, the maximum queue length is achieved, and at this moment, all the densities of 


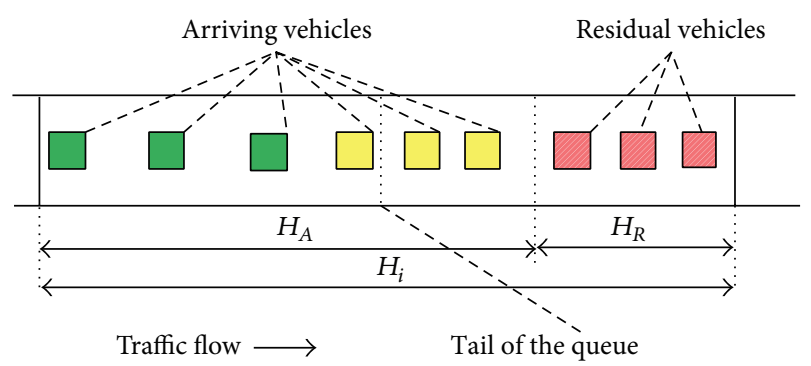

FIGURE 6: Vehicles spatial distribution in queue tail cell.

the downstream of the queue tail cell are equal to the critical density. Therefore, it can be concluded that if the cell $i$ and all its downstream cell densities are larger than the critical density and if the upstream cell densities are lower than the critical density, the queue tail locates in cell $i$ or cell $i-1$.

Further, to analyze whether the queue tail still stays in cell $i$ or moves to cell $i-1$, the demanded space from cell $i-1$ and the supplied space from cell $i$ should be compared. If all the last time interval vehicles of cell $i-1, n_{i-1}(k-1)$, flow into cell $i$ in this time interval, it suggests that the traffic flow is free and the queue tail is still in cell $i$. Otherwise, the tail of the queue is located in cell $i-1$. Thus, it can be summarized that the queue tail is located in cell.

3.2. Method of Queue Length Calculation. To get the exact point where the tail of the queue is located, it is necessary to analyze the distribution of the density in the queue tail cell identified in Section 3.1. The queue tail cell is divided into a number of homogenous sections. In this paper, the queue tail is defined as to where the shockwave propagates. So, the queue length can be estimated as long as the exact space is identified, whose density is $\rho_{b}$. The point $A$ in Figure 2 represents the initial formation of the queue with the increasing density.

The vehicles in the queue tail cell can be divided into three categories, according to the difference in densities. The rules for classification are as follows:

(1) The first category refers to the vehicles that did not move to the downstream cell in the last time interval, since the supply space was not enough. They are shown in Figure 6 as red ones. The density of this section approximately equals its immediate downstream cell $\rho_{i+1}$.

(2) The vehicles of the second category are those that came in the last time interval from the upstream cell; they meet the first category vehicles tail. Due to different braking time of the vehicles in this category, the density $\rho_{\text {trans }}$ is not uniform, and it gradually changes from the density of the first category to the one of the third category vehicles. The vehicles are indicated as yellow ones in Figure 6 .

(3) All other vehicles arrive to the queue tail cell in the last time interval and do not reach the tail of the first category vehicles, so that these vehicles do not have to brake, and their density $\rho_{\text {in }}$ is approximately that of the upstream cell in the last interval. The green ones in Figure 6 represent these vehicles.

According to the analysis above, the density of cell $i+1$ is greater than the critical density $\rho_{b}$ and the density of the third category vehicles is less than $\rho_{b}$. Therefore, there must be a section in the transform area (the second category) with the density equal to $\rho_{b}$, and the location of the queue tail is shown in Figure 6 as the red line. To calculate the queue tail location, the numbers of vehicles in the three categories should be obtained.

The spatial length $H_{R}$, which corresponds to the vehicles that remained after the last time interval, equals the number of such vehicles divided by the density of cell $i+1$, which can be written as

$$
H_{R}=\frac{\rho_{i}(k-1) \cdot H_{i}-q_{i+1}(k-1) \cdot T}{\lambda \cdot \rho_{i+1}(k)} .
$$

The supplied space $H_{A}$ for the vehicles arriving in the current interval is the rest of the length of the queue tail cell. As all the vehicles in cell $i-1$ move into the queue tail cell, the density of the third category vehicles can be written as

$$
\rho_{\mathrm{in}, i}(k)=\frac{n_{i-1}(k)}{H_{i-1}} .
$$

Although the densities of sections in the transform area are not uniform, they can be regarded as the average value of $\rho_{\mathrm{in}, i}(k)$ and $\rho_{i+1}(k)$. First, the supplied space $H_{A}$ is filled with vehicles of categories 1 and 2. Second, the vehicles that have arrived to the queue tail cell in the last time interval consist of two parts:

$$
H_{S}=\frac{\chi_{1} \lambda\left(\rho_{\mathrm{in}}+\rho_{i+1}(k)\right)+2 \chi_{2}}{\lambda^{2}\left(\rho_{\mathrm{in}}^{2}+\rho_{\mathrm{in}} \rho_{i+1}(k)\right)} .
$$

The numbers of vehicles in the two parts can be calculated. The functions can be written as follows:

$$
\begin{aligned}
& \chi_{1}=\lambda \frac{\rho_{\mathrm{in}, i}(k)^{2} H_{A}+\lambda \rho_{\mathrm{in}, i}(k) \rho_{i+1}(k) H_{A}-2 \rho_{\mathrm{in}, i}(k) \rho_{i}(k) \cdot T}{\rho_{i+1}(k)-\rho_{\mathrm{in}, i}(k)}, \\
& \chi_{2}=\frac{q_{i}(k-1) \cdot T \cdot \rho_{i+1}(k)-\lambda \cdot \rho_{\mathrm{in}, i}(k) \cdot\left(\rho_{\mathrm{in}, i}(k) \cdot H_{A}+\rho_{i+1}(k) \cdot H_{A}-\rho_{i}(k) \cdot T\right)}{\rho_{i+1}(k)-\rho_{\mathrm{in}, i}(k)} .
\end{aligned}
$$




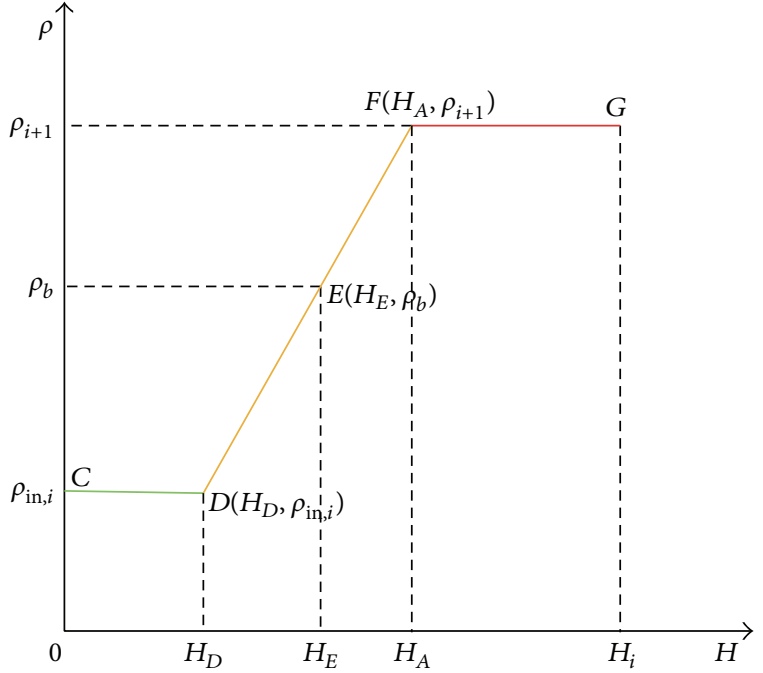

Figure 7: General density distribution in the queue tail cell.

We assume that the densities change from $\rho_{i+1}(k)$ to $\rho_{\text {in }, i}(k)$ linearly in the transform area. For further calculations, the coordinate system is used for the density values and their positions, as shown in Figure 7.

Point $E$ indicates the queue tail position, and its ordinate value is known as $\rho_{b}$. The ordinate value of the line segment FG means the density of the first category vehicles, and point $F$ represents the tail of these vehicles with coordinates $\left(\chi_{1} / \lambda \rho_{\text {in }, i}(k)+2 \cdot \chi_{2} /\left(\lambda \rho_{i+1}(k)+\lambda \rho_{\text {in }, i}(k)\right), \rho_{i+1}(k)\right)$. The line segment $C D$ represents the third category vehicles, and the coordinates of its starting point $D$ are $\left(\chi_{1} / \lambda \rho_{\text {in }, i}(k), \rho_{\text {in }, i}(k)\right)$. To obtain the coordinates of point $E$, its relationship with the known points $F$ and $G$ should be established.

As illustrated in Figure 7, Points $D, E$, and $F$ are located on the same line, with the slope that can be written as

$$
\begin{aligned}
\frac{\chi_{1} / \lambda \rho_{\mathrm{in}, i}(k)+2 \cdot \chi_{2} /\left(\lambda \rho_{i+1}(k)+\lambda \rho_{\mathrm{in}, i}(k)\right)-\chi_{1} / \lambda \rho_{\mathrm{in}, i}(k)}{\rho_{i+1}(k)-\rho_{\mathrm{in}, i}(k)} \\
=\frac{H_{E}-\chi_{1} / \lambda \rho_{\mathrm{in}, i}(k)}{\rho_{b}-\rho_{\mathrm{in}, i}(k)} .
\end{aligned}
$$

The abscissa of point $E$ can be obtained using (15) and (16), as shown below:

$$
\begin{aligned}
& H_{E} \\
& =\frac{\left(\rho_{b}-\rho_{\mathrm{in}, i}(k)\right) 2 \cdot \chi_{2}}{\lambda^{2} \rho_{\mathrm{in}, i}(k)\left(\rho_{i+1}(k)-\rho_{\mathrm{in}, i}(k)\right)\left(\rho_{i+1}(k)+\rho_{\mathrm{in}, i}(k)\right)} \\
& +\frac{\chi_{1}}{\lambda \rho_{\mathrm{in}, i}(k)} \text {. }
\end{aligned}
$$

To avoid zero denominators, three cases should be analyzed according to their physical meanings.

As shown in Figure 8(a), if the density in cell $i+1$ is zero, $\rho_{i+1}(k)=0$, then the signal just switched to red and vehicles begin to accumulate. There are no vehicles in the downstream cell of the output cell. The traffic density of output cell $I$ becomes jam $\rho_{J}$ during the red phase, so it can be assumed that $\rho_{I+1}(k)$ equals $\rho_{J}$ until the green phase begins. In addition, because no vehicles flow into cell $I+1$, the traffic flow from cell $I$ is zero. So, the function to calculate $H_{E}$ can be written as follows:

$$
\begin{aligned}
H_{E}= & \frac{\left(\rho_{b}-\rho_{\mathrm{in}, i}(k)\right) 2 \cdot \chi_{2}}{\lambda^{2} \rho_{\mathrm{in}, i}(k)\left(\rho_{J}-\rho_{\mathrm{in}, i}(k)\right)\left(\rho_{J}+\rho_{\mathrm{in}, i}(k)\right)} \\
& +\frac{\chi_{1}}{\lambda \rho_{\mathrm{in}, i}(k)} .
\end{aligned}
$$

When the input density is zero, $\rho_{\text {in }, i}(k)=0$, it means that there were no vehicles flowing into cell $i$ from cell $i-1$ in the last time interval, $q_{i}(k-1)=0$. According to Figure $8(\mathrm{~b})$, it can be seen that point $E$ does not exist, and the queue tail is represented by point $F$. Therefore, the queue tail is located at the end of the remaining vehicles from last time interval:

$$
H_{E}=H_{R}-\frac{n_{i}(k-1)-q_{i+1}(k) \cdot T}{\lambda \cdot \rho_{i+1}(k)} .
$$

Because the density value cannot be negative, $\rho_{i+1}(k)$ is equivalent to $\rho_{\text {in, } i}(k)$. In addition, as analyzed in Section 3.1, the density of the third category vehicles is equal to or less than the critical density, and the vehicle density in cell $i+1$ is equal to or is greater than $\rho_{b}$. Therefore, if $\rho_{i+1}(k)=\rho_{\text {in }, i}(k)$, then both are equal to the critical density. This indicates that the tail of the queue is located at the boundary of cell $i$ and cell $i-1$, as shown in Figure 8(c).

The queue length $H_{q}(k)$ in a certain short time interval $T$ can be calculated based on the queue tail location and can be written as

$$
H_{q}(k)=\sum_{j=I-i-1}^{I}\left(H_{i}-H_{E}+H_{j}\right) .
$$

In signal cycle $m$, the average queue length $H_{m}^{c a}$ is the arithmetic mean of every queue length in this cycle:

$$
H_{m}^{c a}=\sum_{p=0}^{C / T} H_{q}(k)(m \cdot C+p) .
$$

The maximum queue length in signal cycle is the longest queue in every short time interval.

\section{Implementation}

4.1. Implementation Procedure. Figure 9 emphasizes the idea that the procedure of queue length estimation consists of two major steps. First, traffic flow parameters in all cells are calculated based on the modified CTM. If the density in cell $I$ is larger than the critical density and all the upstream densities are larger than the critical density down to cell $i-1$, it can be judged that the queue tail is located in cell $i$ or cell $i-1$. The exact queue tail cell is identified by verifying whether all the vehicles from cell $i-1$ moved to cell $i$ in the last time interval. Second, the queue tail is located within the cell by transforming the problem into searching of 


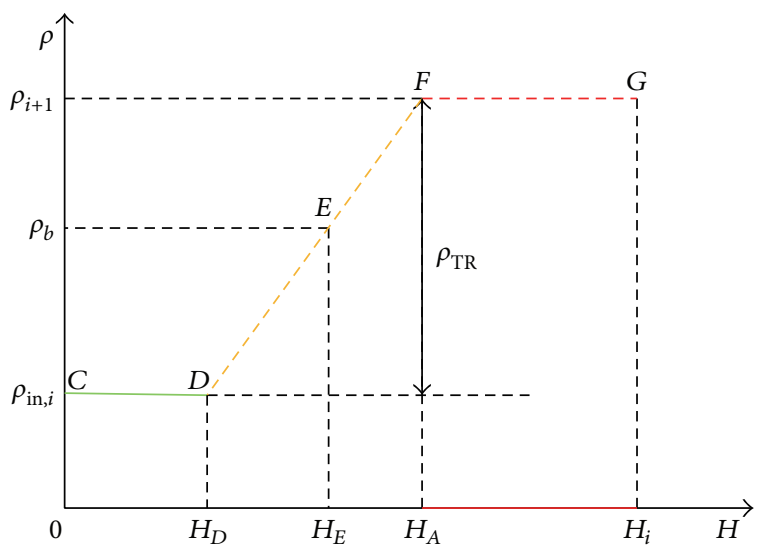

(a) Case 1

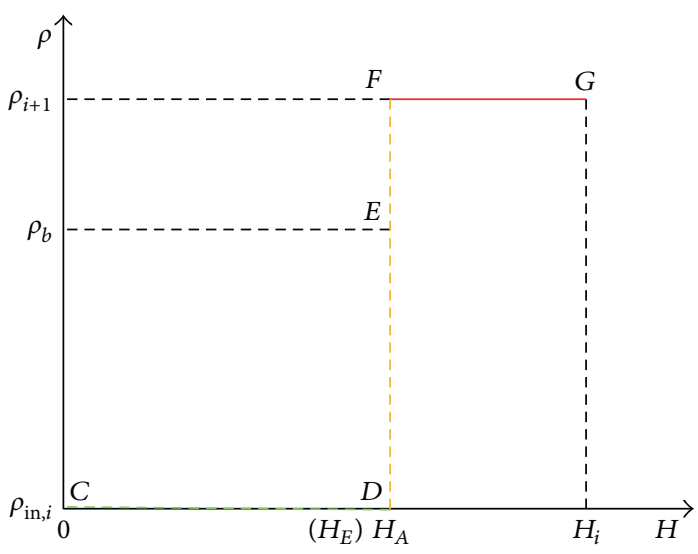

(b) Case 2

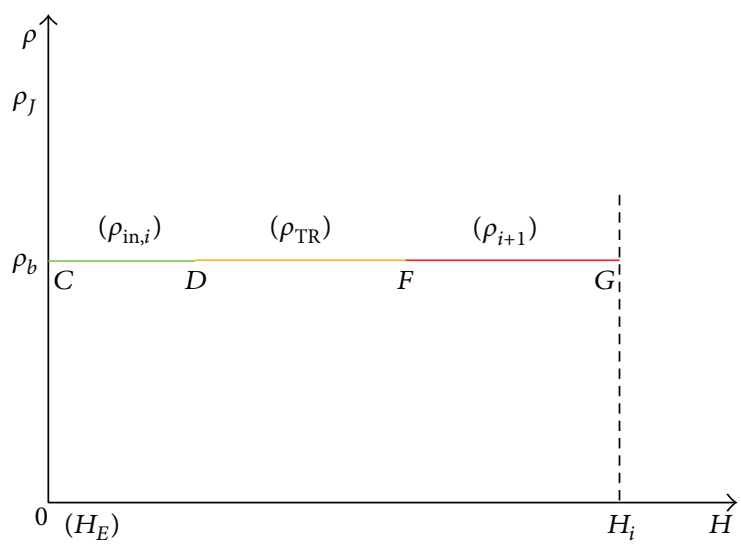

(c) Case 3

FIgURE 8: Density distributions in queue tail cell for three special cases.

the point whose ordinate value is the critical density $\rho_{b}$. Then, the general formula for the queue length in the queue tail cell is deduced. Three special cases mentioned in this paper should be considered in addition to the general estimation of the queue tail. Finally, the queue length in the current time interval can be easily calculated by adding the queue length in the queue tail cell and the lengths of all the cells downstream.

4.2. Simulation Evaluation Based on Field Data. The intersection of Yatai Street and Ziyou Road in Changchun, China, is selected as the investigation site, because they are two arterial roads without much interruption due to secondary roads, and traffic flows include both free and saturated conditions. The actual data of the test road was used for calibrating the related parameters of the simulated traffic environment such as the free-flow speed $54 \mathrm{~km} / \mathrm{h}$, critical density $33.3 \mathrm{veh} / \mathrm{km} / \mathrm{lane}$, jam density $144.4 \mathrm{veh} / \mathrm{km} /$ lane, the distribution of the traffic flow in the test road, and the capacity. Simulation model was verified by comparing the output parameters with the field data collected from the video detector temporally located on the street. The input traffic parameters for simulation software are investigated, including the signal cycle $200 \mathrm{~s}$, green ratio 0.36 , and traffic volume measured every 5 minutes during 35 signal cycles. Figure 10 shows the site of test. The length of the road between the fixed detector and stop line is $480 \mathrm{~m}$. The time interval was chosen to be two seconds. Accordingly, the selected street is divided into 16 sections. VISSIM output includes the second-by-second data collected by the fixed detector, maximum queue length, and average queue length in one signal cycle.

The queue length is calculated by a Matlab program, and the proposed model based on the modified CTM is tested. We also selected two other traditional approaches for comparison. One method is based on the shockwave theory. According to Liu et al. [21], the queue back is the maximum queue length in a signal cycle. We compared the maximum queue length estimated by the proposed model with the shockwave theory. The key of shockwave based method is to identify points where queuing shockwave meets discharging shockwave. The location of the identified point represents the rear of the maximum queue. Therefore, the maximum queue length is obtained. The performance of the two methods is shown in Figure 11. For the evaluation of the average queue length, we selected a statistical model based on the vacation queuing theory $[31,32]$. According to the model, the queuing and discharging processes are regarded as the fixed vacation time following the $M / G / 1$ distribution. The input process follows the passion distribution, service time 


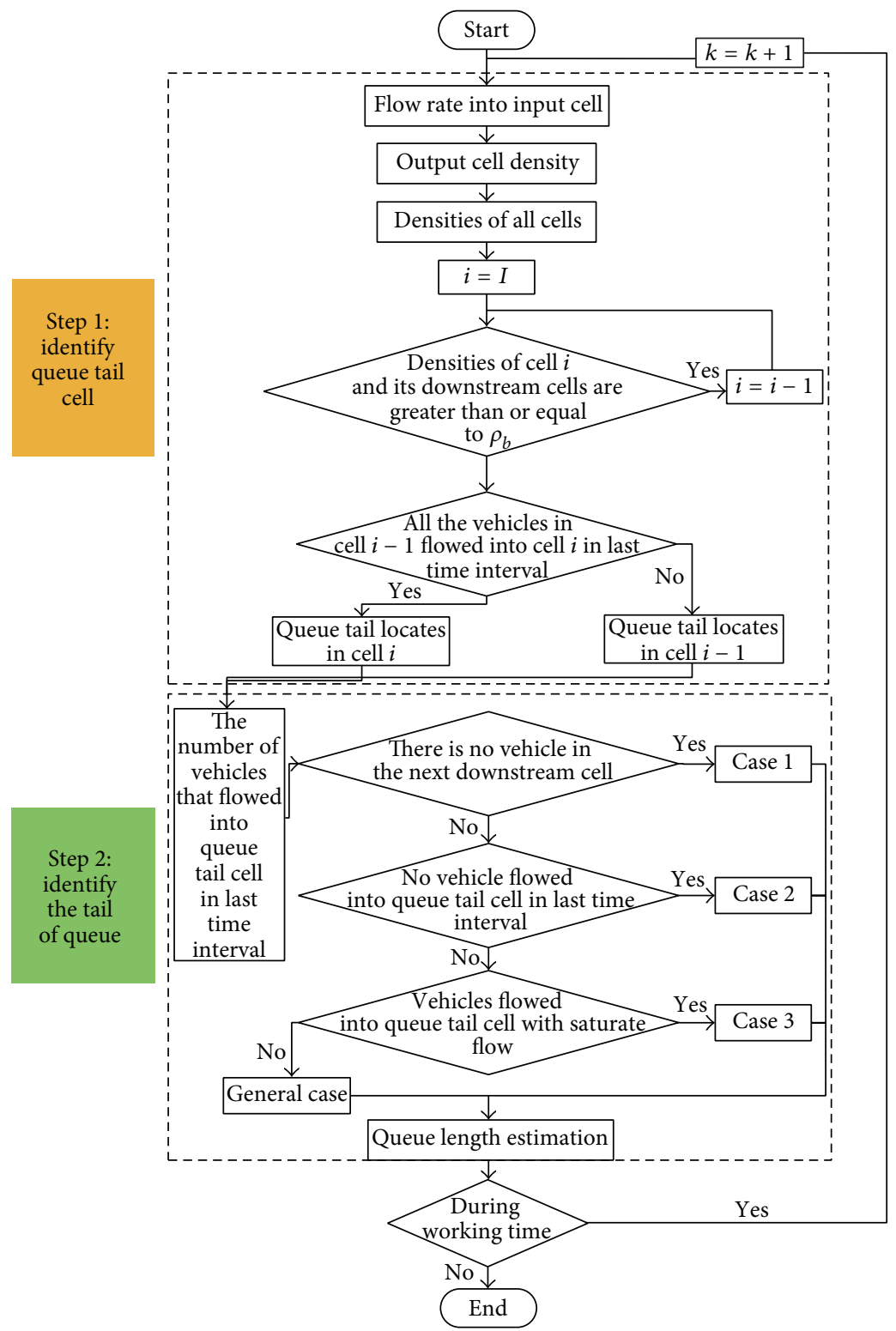

FIGURE 9: Flow chart of the implementation of queue length estimation.

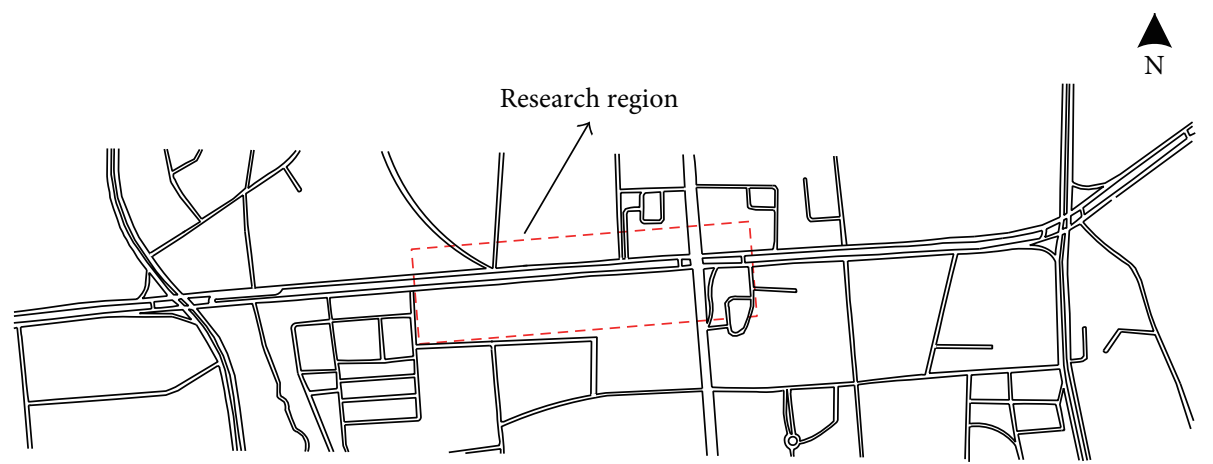

FIGURE 10: Intersection for testing. 


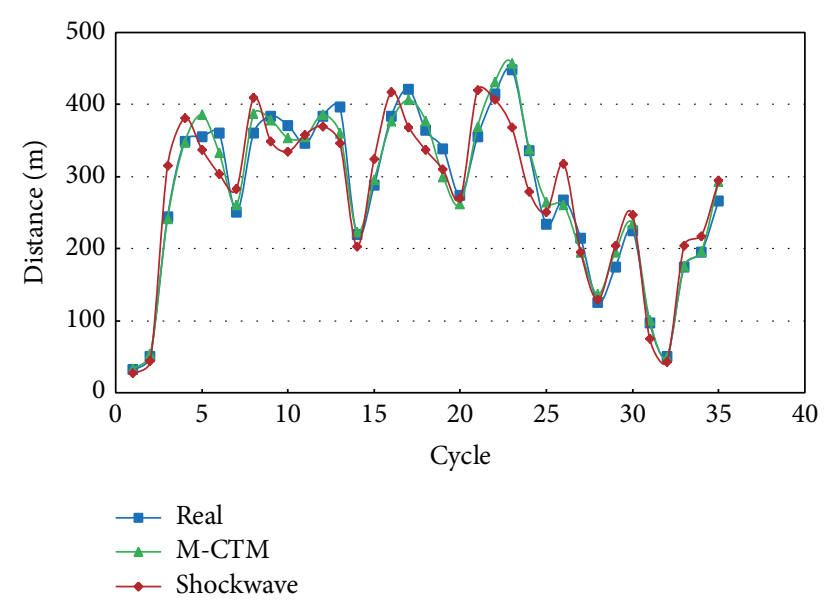

FIGURE 11: Maximum queue length estimation comparison.

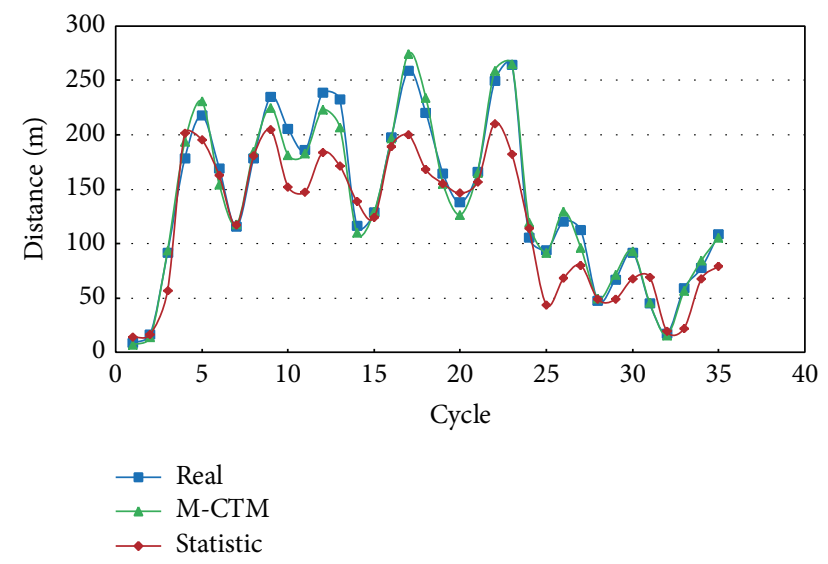

FIGURE 12: Average queue length estimation comparison.

follows the general distribution, and vacation time is the red phase time in a signal cycle. Then, the average queue length is the expected value of the queue length. The performance of our method and the average queue length method is shown in Figure 12. Tables 2 and 3 show the Mean Absolute Errors (MAE) and Mean Relative Errors (MRE) calculated using the following formulas:

$$
\begin{aligned}
& \text { MAE }=\frac{1}{n} \sum_{n} \mid \text { output }- \text { estimation } \mid, \\
& \text { MRE }=\frac{1}{n} \sum_{n}\left|\frac{\text { output }- \text { estimation }}{\text { output }}\right| \times 100 \%,
\end{aligned}
$$

where $n$ is the sample size.

As presented in Table 2, the proposed model based on the modified CTM can estimate the maximum queue length in one signal cycle with the MRE of $4.79 \%$, where the absolute error is about 12.79 meters. As shown in Figure 11, almost all the relative errors are less than $10 \%$. The traditional shockwave based model can estimate the maximum queue with the MRE of $11.59 \%$ and absolute error of about 31 meters. Figure 11 also presented that almost all the relative
TABLE 2: Errors of maximum queue length estimation.

\begin{tabular}{lcc}
\hline & Shockwave based model & M-CTM based model \\
\hline MAE & $30.66 \mathrm{~m}$ & $12.79 \mathrm{~m}$ \\
MRE & $11.59 \%$ & $4.79 \%$ \\
\hline
\end{tabular}

TABLE 3: Errors for average queue length estimation.

\begin{tabular}{lcc}
\hline & Statistic based model & M-CTM based model \\
\hline MAE & $26.25 \mathrm{~m}$ & $7.67 \mathrm{~m}$ \\
MRE & $20.79 \%$ & $6.11 \%$ \\
\hline
\end{tabular}

errors are less than $16 \%$. The comparison results indicate that the proposed model can exactly describe the traffic flow at a signal intersection of an urban road and estimate the maximum queue length with satisfactory accuracy.

Because the average queue length is calculated using the mean queue values of every time interval during one signal cycle, corresponding results can partly represent realtime estimation performance. As indicated in Figure 12, the proposed model also performs well in estimating the average queue length in every signal cycle, with relatively low errors compared to the maximum queue length estimations. The MRE of the proposed estimation model based on the MCTM is about $6 \%$ lower than the traditional statistical model having the MRE of $20.79 \%$. The proposed model performed especially well compared to the traditional method when the traffic flow fluctuations were high.

As shown in Figure 13, the proposed model performs well in describing the queue formation and discharging process for both long and short queues. Here, a short queue is the one when all vehicles can pass the stop line in one signal cycle, and a long queue is the one where some vehicles in the queue need to wait for the next cycle. Overall, the proposed model can successfully estimate the maximum and average queue lengths and succeeds in tracking real-time queue trajectories with satisfactory accuracy.

\section{Discussion and Future Research}

In this paper, we propose the M-CTM based model to estimate the real-time queue length at a signalized intersection, instead of just calculating the maximum or average queue length as traditional methods often do. The queue length in one time interval is estimated in two steps. At the first step, we estimate the densities in all cells based on the M-CTM. The CTM model was modified to describe the traffic flow at a signalized intersection of an urban road. Figure 4 shows the accuracy of the modified CTM in describing the queue forming and discharging processes. At the second step, the density estimated in the first step is analyzed to determine the queue tail position. First, the cell where the queue tail is located is identified. Then, through the construction of the spatial distribution density in the queue tail cell, the exact position of the queue tail is located. There are three special cases, presented in Figure 8, which cannot be calculated using a general formula. Every queue length in a short time interval is calculated; and the queue trajectory is estimated as well. 


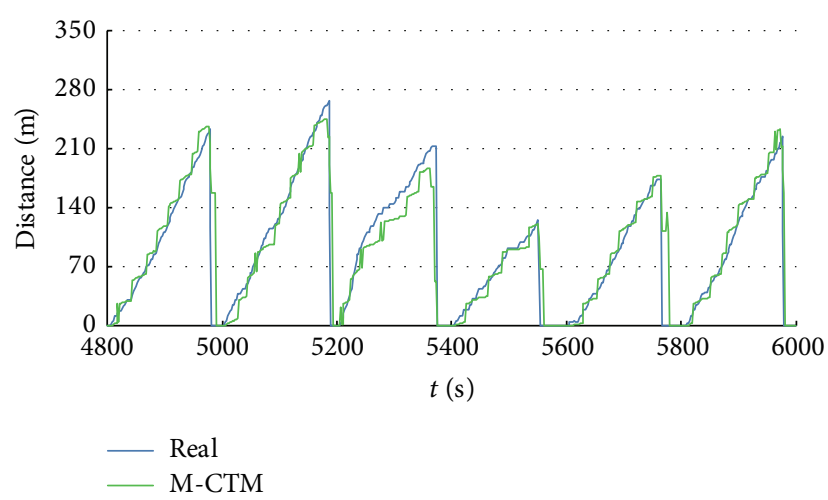

(a) Short queue dynamics estimated

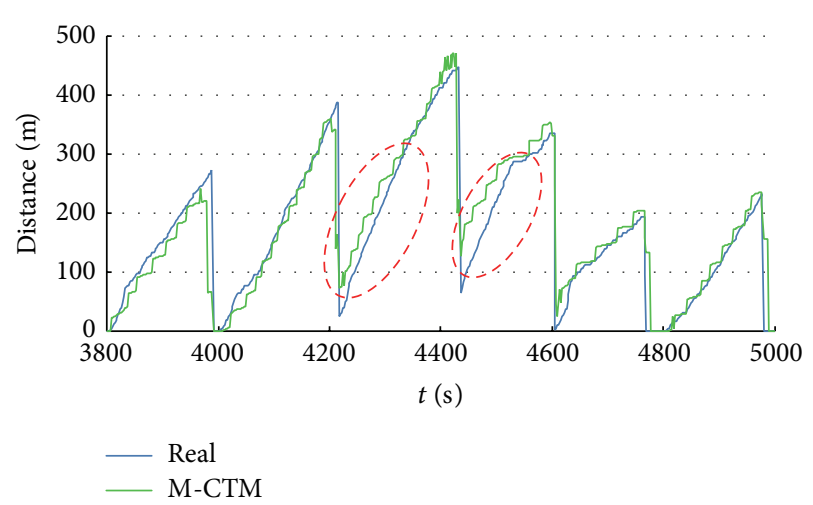

(b) Long queue dynamics estimated

FIGURE 13: Queue trajectory.

The proposed model was tested on VISSIM simulation platform, which was calibrated using field data. The results show the following:

(1) As indicated in Table 1, the traditional CTM can describe the queue forming process for signalized intersections on urban roads; however, the description of the releasing process is not quite accurate. As a basis of the queue length estimation, the exact estimation of the density is necessary. In comparison, Figure 4 shows that the evaluation of the queue discharging process obtained by the proposed modified model is close to the real conditions.

(2) The test results of Section 4.2 suggest that the method proposed in this paper can estimate the maximum and average queue lengths in every signal cycle regardless of whether there is congestion or not. It should be mentioned that, as shown in Figures 11 and 12 , the M-CTM based model performs better than the traditional CTM, especially during a saturated period. It means that the proposed approach is more robust.

(3) Because each time interval is very short, we assume that the queue length estimated in a time interval represents the average length in the current time interval. Every queue length is calculated in short time intervals, second-by-second, to obtain real-time queue length estimations. As can be seen in Figure 13, the proposed model performs well in tracking the queue tail trajectory with satisfactory accuracy, under both free-flow and saturated conditions.

Although the proposed model performs well according to the test results, there are several limitations, which can be addressed in future work:

(1) The proposed model needs further testing and validation and evaluation using field data collected by fixed detectors directly. We will conduct more experiments to test the performance of the proposed model under different traffic conditions. There are some other problems, such as errors caused by detectors.
Therefore, preprocessing of the raw data collected by fixed detectors is generally needed.

(2) In this paper, the vehicles are not allowed to change lanes after passing detectors. However, the distance between detectors and the stop line is long enough, so, in real world situations, drivers have enough time and space to change the lane. Therefore, some methods for taking lane changing in relative publications into account should be considered into the proposed model.

(3) As is indicated in Figure 13(b), highlighted with circles, during $4200-4600 \mathrm{~s}$ the proposed model overestimated the queue length. It means that the error accumulation exists in the proposed model, and the problem is especially critical during congestion periods, because the system has no chance to clear the vehicles and reset the density values.

(4) Although the time interval adopted in testing is very short, the span still exists. The queue trajectory estimated is not as smooth as the real one. A filtering method, such as Kalman filter, should be used to smooth the estimated results, and improve the accuracy of the proposed model.

\section{Conflict of Interests}

The authors declare that there is no conflict of interests regarding the publication of this paper.

\section{Acknowledgment}

The work was supported by the National Natural Science Foundation of China (Grant no. 51378237).

\section{References}

[1] F. V. Webster and B. M. Cobbe, “Traffic signals," Road Research Technical Paper 56, Road Research Laboratory, Her Majesty's Stationery Office, London, UK, 1966. 
[2] W. B. Cronje, "Analysis of existing formulas for delay, overflow, and stops," Transportation Research Record, vol. 905, pp. 89-93, 1983.

[3] K. Balke, H. Charara, and R. Parker, "Development of a traffic signal performance measurement system (TSPMS)," Report 04422-2, Texas Transportation Institute, 2005.

[4] F. Webster, “Traffic signal settings," Road Research Technical Paper 39, Road Research Laboratory, Her Majesty's Stationery Office, London, UK, 1958.

[5] G. F. Newell, "Approximation methods for queues with application to the fixed-cycle traffic light," SIAM Review, vol. 7, no. 2, pp. 223-240, 1965.

[6] D. H. Green, "Control of oversaturated intersections," Journal of the Operational Research Society, vol. 18, no. 2, pp. 161-173, 1967.

[7] P. G. Michalopoulos and G. Stephanopoulos, "Oversaturated signal systems with queue length constraints-I: single intersection," Transportation Research, vol. 11, no. 6, pp. 413-421, 1977.

[8] T.-H. Chang and J.-T. Lin, "Optimal signal timing for an oversaturated intersection," Transportation Research Part B: Methodological, vol. 34, no. 6, pp. 471-491, 2000.

[9] P. B. Mirchandani and Z. Ning, "Queuing models for analysis of traffic adaptive signal control," IEEE Transactions on Intelligent Transportation Systems, vol. 8, no. 1, pp. 50-59, 2007.

[10] A. D. May, "Traffic flow theory-the traffic engineers challenge," in Proceedings of the Institute of Traffic Engineering, pp. 290-303, 1975.

[11] R. Akcelik, A Queue Model for HCM 2000, ARRB Transportation Research, Vermont South, Australia, 1999.

[12] G. Vigos, M. Papageorgiou, and Y. Wang, "Real-time estimation of vehicle-count within signalized links," Transportation Research Part C: Emerging Technologies, vol. 16, no. 1, pp. 18-35, 2008.

[13] N. Geroliminis and A. Skabardonis, "Prediction of arrival profiles and queue lengths along signalized arterials by using a markov decision process," Transportation Research Record, vol. 1934, no. 1, pp. 116-124, 2005.

[14] H. Heffes and D. M. Lucantoni, "Markov modulated characterization of packetized voice and data traffic and related statistical multiplexer performance," IEEE Journal on Selected Areas in Communications, vol. 4, no. 6, pp. 856-868, 1986.

[15] M. Poch and F. Mannering, "Negative binomial analysis of intersection-accident frequencies," Journal of Transportation Engineering, vol. 122, no. 2, pp. 105-113, 1996.

[16] M. J. Lighthill and G. B. Whitham, "On kinematic waves. I. Flood movement in long rivers," Proceedings of the Royal Society of London A, vol. 229, no. 1178, pp. 281-316, 1955.

[17] M. J. Lighthill and G. B. Whitham, "On kinematic waves. II. A theory of traffic flow on long crowded roads," Proceedings of the Royal Society of London A, vol. 229, no. 1178, pp. 317-345, 1955.

[18] P. I. Richards, "Shock waves on the highway," Operations Research, vol. 4, no. 1, pp. 42-51, 1956.

[19] G. Stephanopoulos, P. G. Michalopoulos, and G. Stephanopoulos, "Modelling and analysis of traffic queue dynamics at signalized intersections," Transportation Research Part A: General, vol. 13, no. 5, pp. 295-307, 1979.

[20] A. Skabardonis and N. Geroliminis, "Real-time estimation of travel times on signalized arterials," in Proceedings of the 16th International Symposium on Transportation and Traffic Theory, pp. 387-406, College Park, Md, USA, July 2005.
[21] H. X. Liu, X. Wu, W. Ma, and H. Hu, "Real-time queue length estimation for congested signalized intersections," Transportation Research Part C: Emerging Technologies, vol. 17, no. 4, pp. 412-427, 2009.

[22] C. F. Daganzo, “The cell transmission model: a dynamic representation of highway traffic consistent with the hydrodynamic theory," Transportation Research Part B: Methodological, vol. 28, no. 4, pp. 269-287, 1994.

[23] L. Muñoz, X. Sun, D. Sun, G. Gomes, and R. Horowitz, "Methodological calibration of the cell transmission model," in Proceedings of the American Control Conference, vol. 1, pp. 798803, IEEE, Boston, Mass, USA, June-July 2004.

[24] X. Sun, L. Muñoz, and R. Horowitz, "Mixture kalman filter based highway congestion mode and vehicle density estimator and its application," in Proceedings of the 2004 American Control Conference (AAC '04), pp. 2098-2103, July 2004.

[25] C. M. J. Tampère and L. H. Immers, "An extended Kalman filter application for traffic state estimation using CTM with implicit mode switching and dynamic parameters," in Proceedings of the IEEE Intelligent Transportation Systems Conferenc (ITSC '07), pp. 209-216, IEEE, Seattle, Wash, USA, October 2007.

[26] M. Hadiuzzaman and T. Z. Qiu, "Cell transmission model based variable speed limit control for freeways," Canadian Journal of Civil Engineering, vol. 40, no. 1, pp. 46-56, 2013.

[27] Y. Kim and H. Keller, "On-line traffic flow model applying the dynamic flow-density relation," in Proceedings of the 11th International Conference on Road Transport Information and Control, pp. 141-145, London, UK, March 2002.

[28] T. Pohlmann and B. Friedrich, "Online control of signalized networks using the cell transmission model," in Proceedings of the 13th International IEEE Conference on Intelligent Transportation Systems (ITSC '10), pp. 1-6, IEEE, Funchal, Portugal, September 2010.

[29] B. Xie, M. Xu, J. Härri, and Y. Chen, "A traffic light extension to Cell Transmission Model for estimating urban traffic jam," in Proceedings of the IEEE 24th Annual International Symposium on Personal, Indoor, and Mobile Radio Communications (PIMRC '13), pp. 2566-2570, IEEE, September 2013.

[30] Y.-C. Chiou and Y.-F. Huang, "Stepwise genetic fuzzy logic signal control under mixed traffic conditions," Journal of Advanced Transportation, vol. 47, no. 1, pp. 43-60, 2013.

[31] Y. Levy and U. Yechiali, "Utilization of idle time in an M/G/1 queueing system," Management Science, vol. 22, no. 2, pp. 202211, 1975.

[32] G. Choudhury, "On a batch arrival poisson queue with a random setup time and vacation period," Computers \& Operations Research, vol. 25, no. 12, pp. 1013-1026, 1998. 


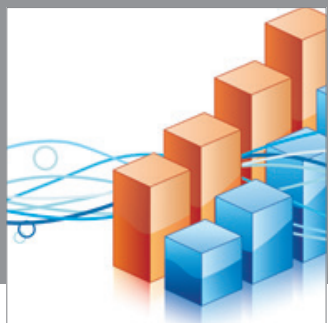

Advances in

Operations Research

mansans

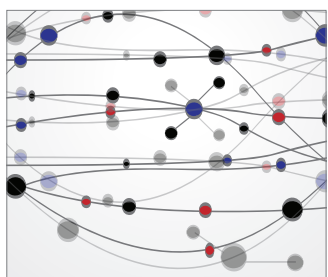

The Scientific World Journal
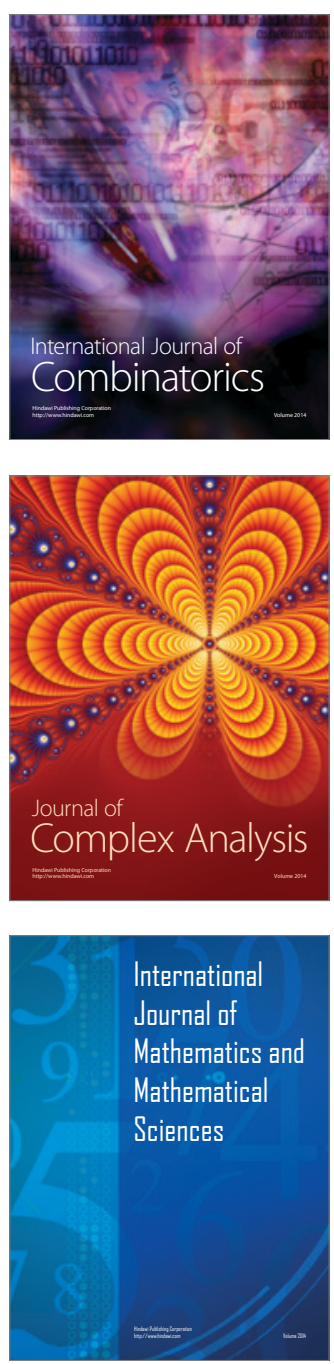
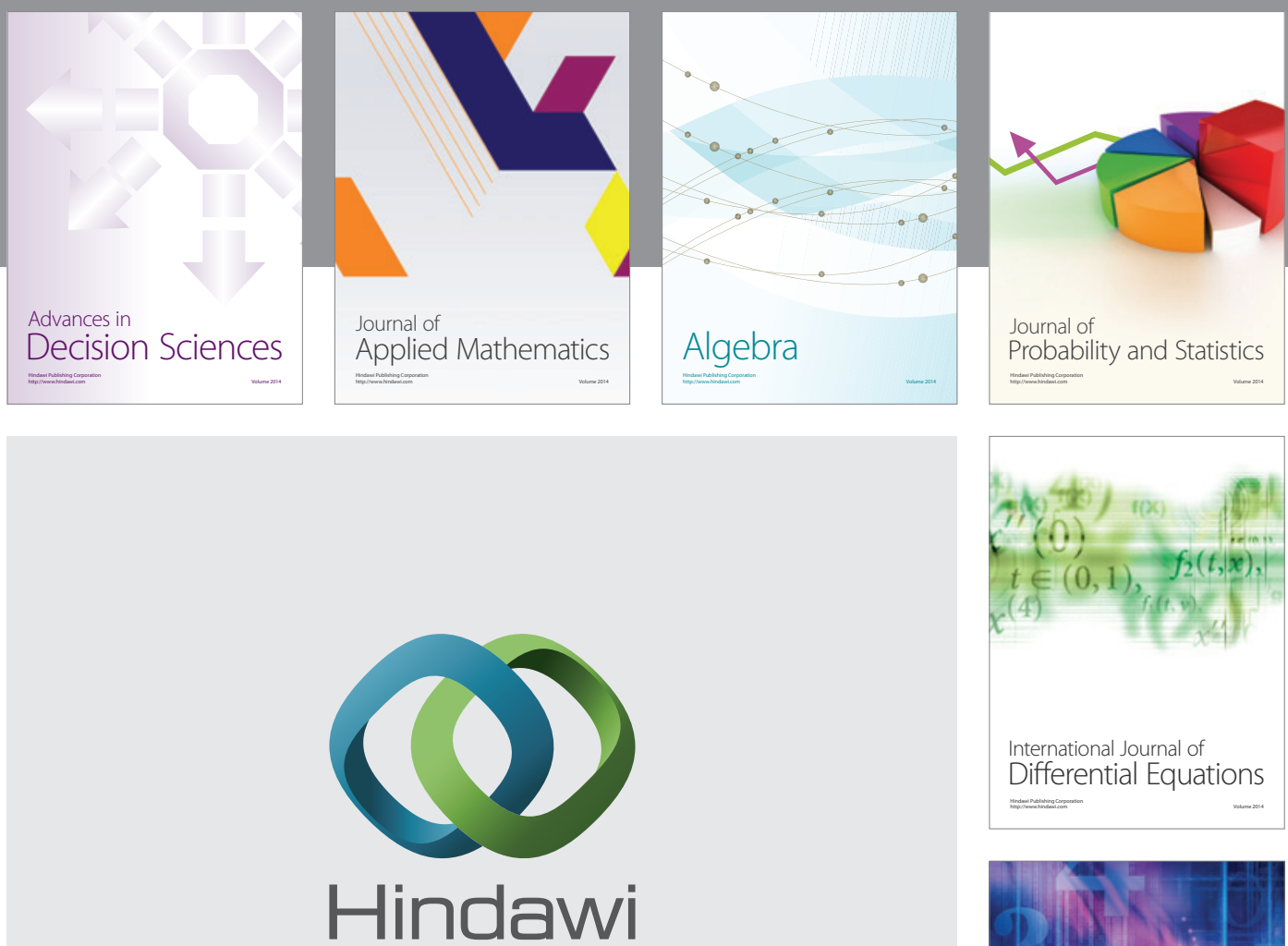

Submit your manuscripts at http://www.hindawi.com
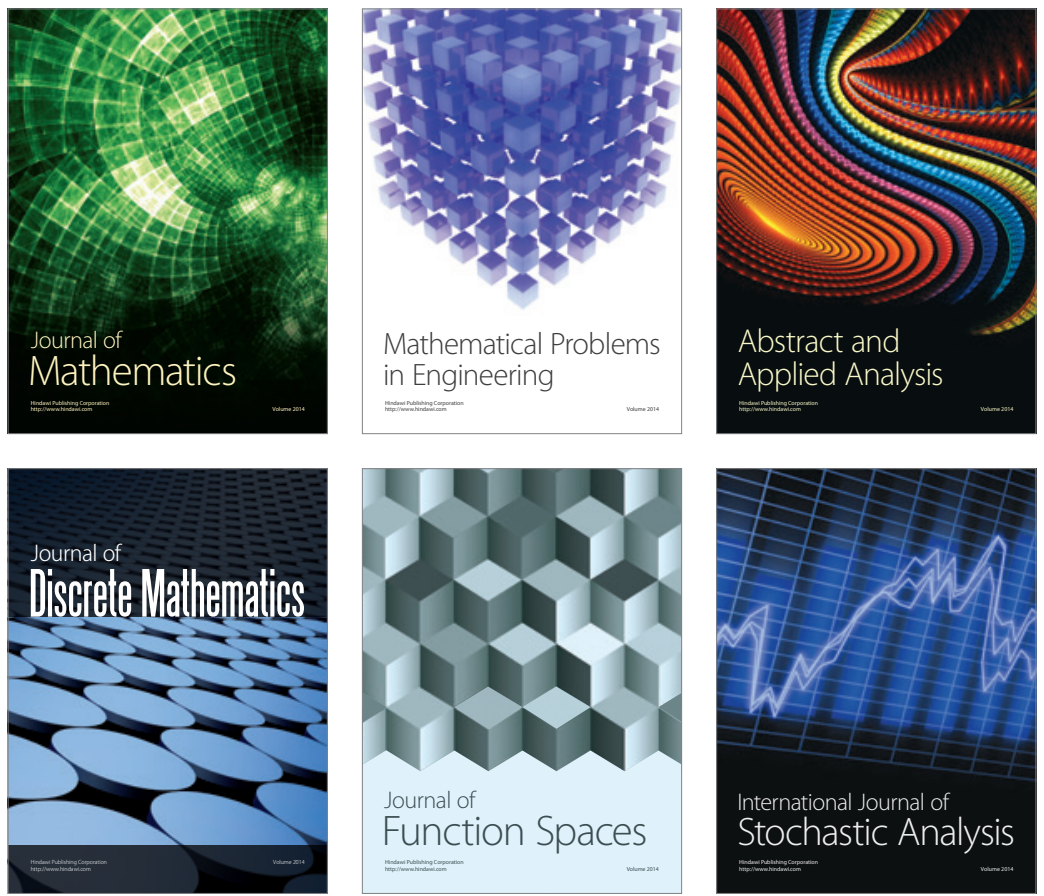

Journal of

Function Spaces

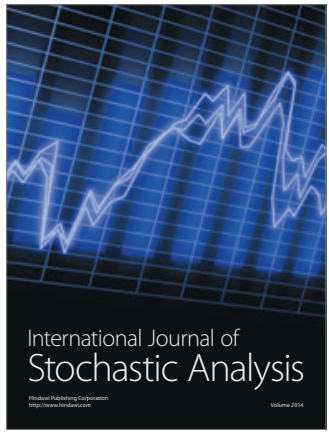

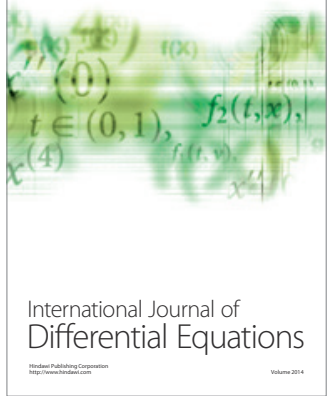
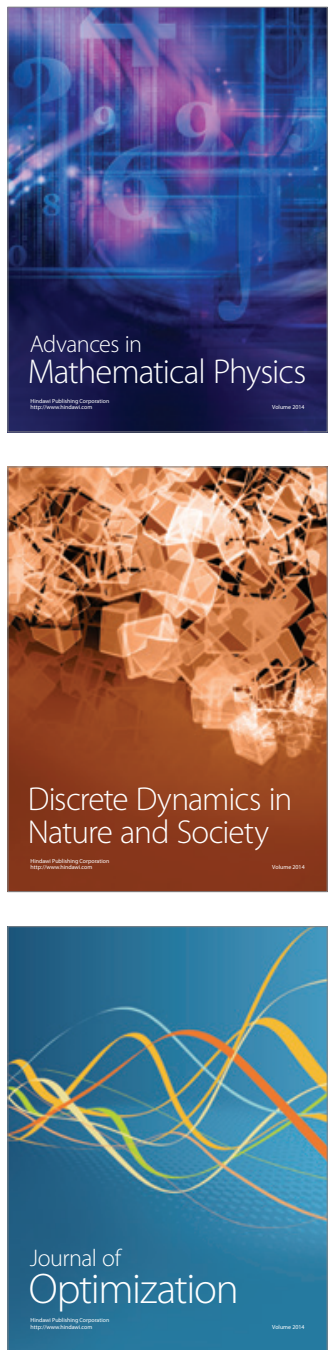